\title{
Elaboração de protocolo de condutas em traqueostomias no hospital referência de tratamento do câncer do Amazonas
}

\section{Elaboration of a tracheostomy conduct protocol in the Amazonas cancer reference hospital}

Maria Carolina Coutinho Xavier Soares, ACBC-AM¹,2; Fernando luiz Westphal, TCBC-AM²; Luiz Carlos de Lima²; Jefferson Moreira Medeiros ${ }^{2}$

\section{R E S U M O}

\begin{abstract}
Objetivo: criar um manual de rotina multidisciplinar de condutas em traqueostomias para pacientes adultos e pediátricos da Fundação Centro de Controle de Oncologia do Estado do Amazonas. Métodos: o protocolo foi desenvolvido por meio do método Delphi modificado, que consistiu na aplicação de duas séries de questionários a 20 profissionais da unidade. Resultados: treze profissionais concluíram as duas etapas. Na primeira etapa, 53 de 92 questões apresentadas obtiveram consenso $(57,6 \%)$. Estas sentenças que obtiveram consenso formaram o texto da segunda etapa, que foi dividido em oito capítulos que foram avaliados por meio da marcação de respostas oferecidas em uma escala Linkert. Todos os capítulos apresentados na segunda etapa obtiveram consenso, significando que a soma das respostas concordo e concordo plenamente foram todas acima de $70 \%$. Conclusão: utilizando os dados obtidos no consenso, foi elaborado um protocolo de condutas em traqueostomias e um manual de orientações de cuidados para os pacientes e seus cuidadores.
\end{abstract}

Descritores: Protocolos Clínicos. Traqueostomia. Oncologia. Hospitais Universitários.

\section{INTRODUÇÃO}

T raqueostomia (TQT) é o procedimento cirúrgico que consiste na abertura da parede anterior da traqueia, comunicando-a com o meio externo através da inserção de uma cânula, tornando a via aérea pérvia1. É um procedimento comum em oncologia, principalmente em oncologia de cabeça e pescoço ${ }^{2}$. Estudos entre os cirurgiões de cabeça e pescoço de vários serviços relataram que 26 a 39\% dos especialistas realizavam o procedimento com frequência, em casos de retalhos e grandes ressecções, de forma preventiva ${ }^{1-3}$. Este procedimento no manejo da via aérea em pacientes oncológicos tem sido indicado também em casos de pacientes com massas tumorais e submetidos à radioterapia prévia, causando obstrução das vias aéreas e impedindo a intubação orotraqueal (IOT) ${ }^{4,5}$. Estudos evidenciam que a média de traqueostomias de rotina em ressecções de carcinoma espinocelular está entre 23 e $30 \%$ e, em casos selecionados, até $74 \% 3,6$

Este procedimento gera inúmeras mudanças no dia a dia do paciente: em sua dinâmica respiratória, em seu comportamento e relacionamento interpessoal, em seu cuidado pessoal. Para que essas mudanças ocorram com melhores resultados, diversos cuidados são necessários. Para todos esses cuidados, observou-se a importância de uma equipe multidisciplinar para o manejo de traqueostomias (médicos, odontólogos, fonoaudiólogos, fisioterapeutas, enfermeiros, nutricionistas) tanto em ambiente hospitalar quanto no seguimento ambulatorial.

\footnotetext{
1 - Fundação Centro de Controle de Oncologia do Amazonas (FCECON), Serviço de Cirurgia de Cabeça e Pescoço, Manaus, AM, Brasil.

2 - Universidade Federal do Amazonas (UFAM), Mestrado Profissional em Cirurgia - PPGRACI, Manaus, AM, Brasil.
} 
$\mathrm{Na}$ Fundação Centro de Controle de Oncologia do Estado do Amazonas (FCECON), observamos a presença de profissionais de inúmeras especialidades atendendo pacientes com TQT, porém ainda não existe uma rotina própria do hospital para adaptar as diretrizes de condutas em traqueostomias à realidade dos nossos pacientes. Não há um modelo uniforme de tratamento do paciente com traqueostomias durante a sua internação, padronização de materiais e procedimentos, nem um modelo de orientações para condutas domiciliares. A elaboração de um manual de rotinas levando em consideração a experiência dos envolvidos nos cuidados com TQTs na FCECON traz subsídio clínico para reprodutibilidade das ações. A apresentação de informações sintetizadas da literatura, elaborada em tópicos para elucidar os questionamentos do dia a dia, mostra-se eficiente no aprimoramento do trabalho em equipe, refletindo em resultados favoráveis em relação à redução de complicações e melhora na qualidade de vida do paciente ${ }^{7}$.

O objetivo deste trabalho foi criar um protocolo multidisciplinar de condutas em traqueostomias para os pacientes adultos e pediátricos da FCECON que necessitarem do procedimento, além de utilizar os dados do protocolo para elaborar um manual de cuidados domiciliares básicos, orientações e condutas de emergência para cuidadores e pacientes.

\section{MÉTODOS}

O projeto foi aprovado pelo Comitê de Ética em Pesquisa da FCECON. CAAE: 61650316.1.0000.0004. Foi utilizado para a elaboração do protocolo de condutas em traqueostomias da FCECON o método Delphi modificado, que consiste em aplicação de séries de questionários a especialistas. Os critérios de inclusão foram: profissionais da FCECON de nível superior que trabalham com pacientes traqueostomizados em sua prática diária; no mínimo cinco anos de experiência na área. Critérios de exclusão: profissionais que por qualquer motivo não completaram as duas etapas do questionário; profissional que perdesse o vínculo de trabalho com a FCECON durante o estudo.

O método Delphi não utiliza cálculo estatístico para definir o número ideal de especialistas para que o consenso seja adequado. Sugere a escolha dos participantes de acordo com o seu conhecimento e experiência no assunto tratado, sempre privilegiando escolher os melhores qualificados do serviço ${ }^{8,9}$. Alguns autores sugerem algumas características básicas para orientar a seleção dos componentes do estudo: especialistas que irão utilizar em sua prática diária os resultados obtidos no protocolo; especialistas que lideram equipes relacionadas ao tema estudado; especialistas considerados como referência em consultas sobre o assunto ${ }^{8}$. Para o presente estudo foram convidados 20 especialistas.

O questionário inicial foi elaborado pela equipe de pesquisadores. As questões do primeiro questionário são produto de revisão de literatura, usando as bases de dados MEDLINE, LILACS. Foram utilizados os termos "tracheostomy" e traqueostomia. Livros textos e protocolos de TQT de outros serviços também foram pesquisados. Foram selecionados 37 textos para a elaboração das sentenças do protocolo.

As variáveis primárias foram definidas como blocos de condutas: indicações, procedimentos, complicações, cuidados de rotina especializado, troca de cânula, decanulação, orientações a pacientes e familiares. As perguntas elaboradas em cada um destes blocos principais foram definidas como variáveis secundárias. O especialista tinha a oportunidade de acrescentar respostas que achasse importante para o protocolo. Ao final de cada questão havia espaço para opiniões, acréscimos e correções. 
Os especialistas selecionados foram convidados a participar do estudo e os que aceitaram assinaram o TCLE. O primeiro questionário foi entregue aos especialistas. A equipe de pesquisa não presenciou preenchimento das respostas. Após o preenchimento a pesquisadora foi acionada e recolheu o questionário, que foi colocado em envelopes em branco, sem identificação. A elaboração de um segundo questionário resultou da análise das respostas apresentadas no primeiro questionário. Todas as respostas que obtiveram acima de $65 \%$ de consenso foram colocadas no texto do segundo questionário. Questões relevantes que não apresentaram consenso no primeiro questionário foram repetidas na segunda etapa.

O segundo questionário transformou as respostas que obtiveram consenso em um texto. O texto foi dividido em oito capítulos para melhor análise. Após a leitura de cada capítulo, o especialista relatava a sua impressão assinalando um item da escala Linkert citada a seguir: 1. Discordo fortemente; 2. Discordo; 3. Neutro; 4. Concordo; 5. Concordo plenamente. O segundo questionário foi entregue ao especialista, seguindo o mesmo protocolo de preenchimento da primeira etapa. Após a análise destas respostas do segundo questionário, os capítulos que obtiveram as respostas concordo e concordo plenamente acima de $70 \%$ de consenso integraram o texto final do protocolo. Utilizando os dados do protocolo, foi elaborado também manual para o paciente.

A estatística descritiva foi calculada para item de cada questão. Foi utilizado cálculo de porcentagem entre as respostas consideradas válidas $^{8}$. O cálculo de $\mathrm{P}$ não se aplica neste tipo de projeto ${ }^{9}$. Foi utilizado o programa Exce/ para registro de dados. Consenso e manual do paciente foram entregues à diretoria clínica do hospital.

\section{RESULTADOS}

Os 20 profissionais de saúde convidados e que aceitaram participar do estudo da FCECON eram de diversas especialidades: médico intensivista, médico da urgência oncológica, fisioterapeuta, enfermeira intensivista, cirurgião oncológico, cirurgião de cabeça e pescoço, cirurgião torácico, enfermeiro do centro cirúrgico, enfermeiro estomatoterapeuta, enfermeiro da endoscopia. Treze (65\%) profissionais participaram das duas séries de questionários e somente esses foram analisados no protocolo. O perfil dos participantes que completaram todas as etapas do projeto pode ser visto na tabela 1.

Tabela 1. Perfil dos profissionais que participaram integralmente das séries de questionários do protocolo de traqueostomia.

\begin{tabular}{lc}
\hline Formação do profissional & $\begin{array}{c}\text { Número de } \\
\text { profissionais }\end{array}$ \\
\hline Cirurgião pediátrico & 1 \\
Cirurgião de cabeça e pescoço & 5 \\
Cirurgião oncológico & 3 \\
Enfermeiro estomatologista & 1 \\
Enfermeiro centro cirúrgico & 2 \\
Enfermeiro endoscopia & 1 \\
\hline
\end{tabular}

O tempo de retorno dos questionários preenchidos na primeira etapa variou de dois a 90 dias. Noventa e duas questões foram apresentadas na primeira etapa, dez questões discursivas e 82 objetivas. Foi facultado ao especialista assinalar quantas sentenças julgasse corretas nas questões objetivas. Em todas as questões objetivas, havia a possibilidade do especialista assinalar uma opção na qual o mesmo não responde à pergunta por não fazer parte da sua prática diária. Quando essa alternativa estava assinalada, a questão deste especialista foi descartada ao realizar a quantificação de respostas 
válidas. Havia espaço para adicionar comentários sobre assuntos relevantes. Após a análise dos questionários preenchidos, 53 questões tiveram $65 \%$ de consenso (Tabela 2). Doze comentários de assuntos que não estavam no texto inicial foram adicionados.

Tabela 2. Resultado da primeira etapa do método Delphi.

\begin{tabular}{lc}
\hline Perfil das questões & Número de questões \\
\hline $\begin{array}{l}\text { Questões apresentadas } \\
\begin{array}{l}\text { Questões selecionadas } \\
\text { por consenso }\end{array}\end{array}$ & 92 \\
\hline
\end{tabular}

Utilizando os dados de consenso da primeira etapa, foi elaborado o texto da segunda etapa a ser apresentado aos profissionais. Seis questões da primeira etapa foram reapresentadas integralmente, por terem sido consideradas relevantes para o protocolo, porém sem consenso (abaixo de 65\%). O texto da segunda etapa foi dividido em oito capítulos para serem avaliados de forma mais clara: conceitos gerais, indicações, procedimento, técnica, complicações, cuidados, decanulação e cuidadores. O tempo de entrega do questionário respondido da segunda etapa variou de um a 21 dias. No capítulo geral, 30,8\% dos especialistas respondeu concordo e 69,2\%, concordo plenamente. No capítulo indicações, $38,5 \%$ dos especialistas respondeu concordo e $53,8 \%$, concordo plenamente. Responderam neutro 7,7\%. No capítulo procedimento, 38,5\% dos especialistas respondeu concordo e 61,5\%, concordo plenamente. No capítulo técnica, 53,8\% dos especialistas respondeu concordo e 23,1\%, concordo plenamente. Responderam neutro 15,4\% e discordo 7,7\%. No capítulo complicações, 61,5\% dos especialistas respondeu concordo e 38,5\%, concordo plenamente. No capítulo cuidados, 46,2\% dos especialistas respondeu concordo e 53,8\%, concordo plenamente. No capítulo decanulação, $38,5 \%$ dos especialistas respondeu concordo e $53,8 \%$, concordo plenamente. Responderam neutro 7,7\%. No capítulo cuidadores, 46,2\% dos especialistas respondeu concordo e 53,8\%, concordo plenamente (Tabela 3). Analisando as seis questões reapresentadas, apenas uma questão atingiu $70 \%$ de aceitação para o consenso.

Foram selecionados para integrar o protocolo os textos que apresentaram acima de $70 \%$ de consenso pelos especialistas por meio da soma das assertivas concordo e concordo plenamente. Durante a redação final do protocolo, optou-se por descrever, no texto final, as questões que não obtiveram consenso entre os especialistas utilizando os dados que já são consenso na literatura, pois os mesmos têm grande importância e não poderiam ser descartados da redação final do protocolo.

Com os dados resultantes do consenso foi redigido um protocolo em traqueostomias para a equipe do hospital e um manual para o paciente e seus cuidadores com orientações gerais (Tabela 4).

\section{DISCUSSÃO}

Mitchell, em 2013, elaborou um consenso de TQTs pelo método Delphi, com 110 questões iniciais. Ao final da segunda etapa, 77 sentenças foram aprovadas e 36 descartadas. Neste estudo nove especialistas participaram da elaboração do consenso, cujas especialidades eram: cirurgiões adultos e pediátricos, enfermeiros, fisioterapeutas e médicos de urgência ${ }^{10}$. Outro consenso publicado sobre TQT utilizou como método reunir profissionais especialistas (19 profissionais) que realizaram uma revisão conjunta da literatura de 40 artigos e, a partir destes dados, redigiram o consenso. Estes profissionais também buscaram adaptar a literatura a sua realidade local. Também foi realizado apenas por médicos e enfermeiros ${ }^{11}$.

Quanto ao presente trabalho, não houve consenso entre os profissionais a respeito do tempo de intubação orotraqueal ideal para indicar traqueostomia. Estudos recentes advogam 
Tabela 3. Resultado da segunda etapa do método Delphi.

\begin{tabular}{lcccc}
\hline $\begin{array}{l}\text { Capítulo do texto/Respostas } \\
\text { positivas dos profissionais }\end{array}$ & $\begin{array}{c}\text { Concordo } \\
\text { plenamente }\end{array}$ & Concordo & Neutro & Discordo \\
\hline Geral & $69,2 \%$ & $30,8 \%$ & & \\
Indicações & $53,8 \%$ & $38,5 \%$ & & \\
Procedimento & $61,5 \%$ & $38,5 \%$ & $15,4 \%$ & $7,7 \%$ \\
Técnica & $23,1 \%$ & $53,8 \%$ & & \\
Complicações & $38,5 \%$ & $61,5 \%$ & & \\
Cuidados & $53,8 \%$ & $46,2 \%$ & $7,7 \%$ & \\
Decanulação & $53,8 \%$ & $38,5 \%$ & & \\
Cuidadores & $53,8 \%$ & $46,2 \%$ & & \\
\hline
\end{tabular}

Tabela 4. Resumo do protocolo obtido por meio das respostas dos especialistas.

\begin{tabular}{ll}
\hline Principais & - Obstrução de vias aéreas altas por neoplasias ou edema ocasionado por radioterapia. \\
indicações de & - Estenoses laringo-traqueais. \\
traqueostomia & - Profilaxia de obstrução de vias aéreas em pós-operatório de cirurgias extensas de cabeça e \\
na FCECON & pescoço.
\end{tabular} sete e 15 dias de intubação orotraqueal, sem possibilidade de desmame da ventilação neste período. Crianças: é possível aguardar até quatro semanas, programando procedimento precoce se não houver previsão de resolução da causa.

- Via aérea difícil.

Procedimento - Explicar previamente ao paciente e familiares sobre o procedimento, apresentar o termo de consentimento para assinatura. Uma cópia fica em posse dos familiares e a outra cópia do termo deve ficar no prontuário médico.

- Definir o local do procedimento: a traqueostomia é um procedimento realizado de rotina no centro cirúrgico. Em situações especiais pode ser realizada em UTI. Pode ser realizado à beira do leito em caso de emergências sem possibilidade de remoção. Traqueostomia no centro cirúrgico, não mobilizando o paciente para a mesa cirúrgica, fazendo o procedimento no leito do paciente: é possível em casos especiais (pacientes obesos mórbidos, em casos em que a mobilização do leito oferece riscos, como fraturas).

- Equipe para o procedimento: cirurgião, auxiliar, circulante, anestesista, enfermeira.

- Traqueostomia sob anestesia geral: paciente já em IOT, em crianças todas as traqueostomias são realizadas preferencialmente sob anestesia geral. Traqueostomia sob anestesia local: paciente com obstrução de vias aéreas, sem possibilidade de IOT.

- Materiais necessários para uma traqueostomia: equipamento para monitorização dos sinais vitais (pressão arterial, ECG, $\mathrm{Spo}_{2}$, capnógrafo), foco cirúrgico, óculos de proteção, avental cirúrgico, campos cirúrgicos, luva estéril, antissépticos, pinça Kelly, tesoura curva, Farabeuf, bisturi elétrico, pinça anatômica, pinça dente de rato, cabo de bisturi no 3 e lâmina 15, porta agulha, fio de sutura nylon 3.0, sonda de aspiração, aspirador, cânula de traqueostomia: ao realizar uma traqueostomia em adultos avaliar a disponibilidade da cânula escolhida (plástica com balonete, plástica sem balonete, metálica). Providenciar sempre o número da cânula estimado para o paciente, além de uma cânula um tamanho acima e uma cânula um tamanho abaixo.

- Tamanhos de cânula, considerações: o diâmetro da cânula de traqueostomia deve ocupar aproximadamente $2 / 3$ ou $3 / 4$ da luz traqueal, definindo o tamanho final ao visualizar a traqueia. Deve-se avaliar como critério o comprimento da cânula em pacientes obesos. 


\section{Continuação Tabela 4.}

Técnica

- A posição preferencial do paciente: decúbito dorsal horizontal, em hiperextensão cervical, utilizando coxim entre as escápulas e rodilha na cabeça. Avaliar sempre se não há contraindicações para este posicionamento.

- Em caso de impossibilidade de hiperextensão cervical, pode ser realizada em decúbito dorsal, em posição neutra. A equipe deve estar preparada para uma dificuldade técnica maior neste procedimento.

- A antissepsia deve ser feita da borda anterior da mandíbula até o terço superior do tórax. Lateralmente até as bordas laterais dos músculos esternocleidomastoideos.

- A incisão da pele em adultos pode ser transversa ou longitudinal. A escolha da incisão deve ser do cirurgião, de acordo com cada paciente. Em crianças a incisão preferencial é a transversa.

- Após o afastamento dos músculos esterno hioide e esterno tireoideo, identificamos o istmo da tireoide. O afastamento cranial do istmo sempre que possível é indicado. Há possibilidade de istmotomia quando necessário.

- Evitar dissecção lateral à traqueia (evitar lesão de laríngeo recorrente, jugular interna, nervo vago, carótida).

- Evitar dissecção inferior (evitar lesão de tronco braquicefálico e inominada).

- A abertura da traqueia deve ser feita idealmente entre o segundo e quarto anéis traqueais.

- Identificação prudente dos anéis traqueais para evitar lesão de cartilagem cricoide e primeiro anel traqueal.

- A técnica utilizada na traqueotomia é definida pelo cirurgião de acordo com as necessidades do paciente: abertura transversa entre os anéis traqueais, ressecção da porção anterior do anel traqueal, ressecção de retalho superior e lateral mantendo boda inferior fixa (retalho de Bjork). Em crianças é indicada a abertura transversa entre os anéis traqueais.

- Em casos de traqueostomia considerada difícil, a sugestão de abordagem técnica é a colocação de fios de reparo na traqueia, exteriorizando para a pele, com o objetivo de identificação do trajeto em casos de decanulação.

- Quando em crianças, utilizar fios de reparo na traqueia de rotina, mesmo em traqueostomias sem dificuldades técnicas, pois o reposicionamento em decanulações acidentais sempre são mais difíceis nesta faixa etária.

- Ao posicionar a cânula de traqueostomia, deve-se ter cuidado para não lesar a parede posterior da traqueia.

- A avaliação do correto posicionamento da cânula após a sua passagem é feita por capnografia ou ausculta pulmonar.

- A fixação da cânula após o posicionamento na traqueia é através de fitas ao redor do pescoço.

- O curativo inicial deve ser feito utilizando gaze ao redor da cânula. 


\section{Continuação Tabela 4.}

Complicações - Materiais considerados indispensáveis para atendimento de complicações das traqueostomias. Devem estar à disposição em setores do hospital onde há permanência de pacientes com traqueostomia, setores de internação, UTI e urgência: cânulas de traqueostomia de todos os tamanhos (em enfermaria de adultos: 5-0, 5-5, 6-0, 6-5, 7-0, 7-5, 8-0, 8-5 em enfermaria pediátrica 3-0, 3-5, 4-0, 4-5, 5-0, 5-5), mangueira de aspiração, sonda de aspiração (em enfermaria adulto : $8,10,12,14$; em enfermaria pediátrica : 4, 6, 8), aspirador, kit de pequena cirurgia, monitorização, fonte de oxigênio, AMBU.

- Decanulação acidental: chamar ajuda de profissional com experiência; se não houver contraindicação, colocar um coxim entre as escápulas e tentar reposicionar a cânula; se não houver possibilidade de uso de coxim, tentar reposicionar em posição neutra; se houver fios de reparo, fazer uma tração leve no mesmo e reposicionar a cânula; em caso de falha de reposicionamento, checar se há na unidade um profissional com experiência e chamar ajuda novamente; monitorização e fonte de oxigênio; aproximar todos os materiais de urgência acima citado.

- Sangramento pós traqueostomia: avaliação inicial pelo médico que estiver disponível no momento se o cirurgião não estiver alcançável de imediato; avaliação precoce do cirurgião; avaliar se o sangramento é originado ao redor da cânula ou do seu interior; providenciar monitorização e oxigenar o paciente; aspirar a cânula em caso de sangramento originado no interior da cânula; avaliar o prontuário do paciente se o mesmo está utilizando medicamentos anticoagulantes.

- Insuficiência respiratória por rolha de secreção na cânula: suspeitar caso o paciente com traqueostomia apresentar dificuldades respiratórias; se o paciente estiver com cânula de plástico, aspirar a cânula; não havendo melhora ou apresentar resistência na passagem da sonda ao tentar aspirar a cânula, nebulizar o paciente com soro fisiológico 0,9\% e tentar novamente a aspiração; se o paciente estiver com cânula metálica, remover a cânula interna imediatamente e proceder à limpeza da mesma; se não houver melhora do padrão respiratório após aspiração e limpeza, é necessário realizar a troca da cânula. Se não tiver treinamento em troca de cânula, chamar ajuda; providenciar monitorização e fonte de oxigênio imediatamente, enquanto se chama ajuda. Disponibilizar a instalar os materiais da lista de urgência.

- Pneumotórax e pneumomediastino pós-procedimento: realizar radiografia de tórax após o procedimento, em casos de dispneia; em crianças a radiografia de tórax é realizada de rotina em todas as traqueostomias; se houver pneumotórax, realizar drenagem fechada de tórax em selo d'água.

- Fístula tráqueo-cutânea: é a epitelização do trajeto do orifício da traqueia até a pele, que permanece pérvio após a retirada da cânula; clinicamente diagnosticado pela queixa do paciente pela persistência de saída de ar e secreção pelo orifício da traqueostomia, após a retirada da cânula; avaliação com o cirurgião para correção da fístula.

- Fístula tráqueo-esofágica: pode-se suspeitar quando há saída de alimento pelo interior da cânula de traqueostomia; pode-se suspeitar quando há pneumonias de repetição; broncoscopia e endoscopia digestiva alta são indicadas quando há suspeita diagnóstica.

- Disfagia pós traqueostomia: se o paciente estiver com cânula com balonete, avaliar se o mesmo não está hiperinsuflado; verificar se há aspiração laringo-traqueal associada; avaliação da fonoaudiologia. 


\section{Continuação Tabela 4.}

Cuidados de rotina e condução do paciente com traqueostomia
- Cuidados com a cânula metálica: retirar a cânula interna; a limpeza da cânula interna pode ser realizada em água corrente, com auxílio de uma escovinha, desde que a água encanada esteja com aspecto transparente e sem resíduos; pode ser usado sabão neutro nesta limpeza, sendo que todo o resíduo deve ser retirado; após limpeza da cânula, secar antes de ser reposicionada; frequência diária de limpeza: definir frequência de limpeza de acordo com o grau de expectoração do paciente.

- Umidificação da traqueostomia: a presença ou não de crostas e a fluidez da secreção durante a aspiração da cânula pode ajudar a medir o grau de umidificação das vias aéreas; de acordo com a fluidez e quantidade de secreção, deve-se definir a frequência de nebulizações do paciente.

- Aspiração da cânula. Equipamentos de proteção para o profissional que irá realizar o procedimento: luva estéril, máscara e óculos de proteção, jaleco ou avental descartável; informar o procedimento ao paciente; todo paciente com traqueostomia deve ter um mecanismo de aspiração próximo ao seu leito (portátil ou vácuo na parede); cateter de aspiração estéril descartável, tamanho selecionado de acordo com o diâmetro da cânula; soro fisiológico para limpeza do cateter de aspiração; ao realizar a aspiração, introduzir o cateter somente até o final da cânula.

- Curativo da traqueostomia: limpeza com soro fisiológico; uso de gaze nas laterais da cânula continuamente; uso de espuma e hidrocoloide a serem avaliados pela equipe de enfermagem; frequência sugerida mínima para curativo ao redor da traqueostomia: 1x ao dia; observar diariamente se há hiperemia na pele ao redor da traqueostomia e se há drenagem de secreções e o aspecto.

- Troca de cânula de traqueostomia: luva estéril, máscara, óculos de proteção, jaleco ou avental descartável para o profissional que irá realizar a troca; material para aspiração disponível; uma cânula do mesmo tamanho e uma um número menor; material para curativo; informar o procedimento ao paciente; remover curativos e roupas que possam bloquear o campo de visão; coxim nas escápulas se não houver contraindicação para hiperextensão cervical; uso de lidocaína gel na porção da cânula a ser introduzida; fixação com fita justa no pescoço; se não houver sucesso na passagem de uma cânula do mesmo número, tentar uma segunda vez com uma cânula menor e se bem-sucedida encaminhar paciente para avaliação do cirurgião; verificação do posicionamento correto da cânula após a troca: paciente mantendo padrão respiratório normal, saída de ar pelo orifício interno da cânula; se a segunda tentativa com a cânula menor não obtiver sucesso, providenciar uma fonte de $\mathrm{O}_{2}$ para o paciente, monitorização; uma segunda pessoa presente deve chamar ajuda em caso de falha na troca; frequência de troca de cânula metálica: a cada 30 dias; frequência de troca da cânula plástica: em até 14 dias.

- Avaliação da possibilidade de fonação do paciente após a traqueostomia: avaliação da fonoaudiologia.

- Decanulação: deve ser particularizada para cada paciente na FCECON, de acordo com a sua doença de base e o estadiamento; avaliar se há programação de procedimentos com anestesia nos próximos sete a dez dias; resolução do motivo que levou o paciente a traqueostomia; paciente consciente; estabilidade hemodinâmica; ausência de estenose traqueal ou glótica; não há sinais de aspiração laringo-traqueal; todos os pacientes devem receber avaliação fonoaudiológica antes do processo de decanulação; em crianças, a broncoscopia é necessária para a decanulação; após a decanulação, há necessidade de seguimento do paciente devido ao aparecimento de complicações precoces e tardias após retirada (sangramento, fístulas, estenoses). 
o procedimento precoce, associando aumento de mortalidade à maior dificuldade de desmame ventilatório, quando associados às traqueostomias tardias (acima de 14 dias após a intubação) ${ }^{12}$. Quando realizada em até sete dias, reduz-se o tempo de permanência na UTI ${ }^{13}$.

Com relação à prevenção de decanulação acidental em traqueostomias consideradas difíceis, não houve consenso sobre como conduzir. Estudos já sugerem realizar reparos na traqueia utilizando fios durante os primeiros dias até a cicatrização do trajeto $^{14}$. A respeito da abertura na traqueia, não houve uma conduta uniforme entre os especialistas. Protocolos mostram que todas as possibilidades de abertura da traqueia têm suas indicações e complicações, realmente não havendo um consenso ${ }^{14,15}$. Em crianças, no entanto, é sugerido apenas incisão longitudinal da traqueia com uso de reparo de fios ${ }^{16}$. A conduta em casos de decanulação acidental não obteve consenso, mesmo sendo reapresentada na segunda etapa. A conduta nos algoritmos de urgências em traqueostomias sugere em casos de decanulação acidental verificar se o paciente está respirando, desinsuflar o balonete, chamar ajuda. É possível tentar reposicionar uma única vez ${ }^{17}$. É de extrema importância que este item esteja relatado em um manual de rotinas, pois é uma das principais causas de morte por complicações de traqueostomia.

Outro item de grande importância que não obteve consenso foram as condutas em casos de obstrução da cânula. O que se observa na literatura é verificar, inicialmente, se o posicionamento da cânula está correto, avaliar se o paciente está respirando, estimular o paciente a tossir, aspirar a cânula, em casos de cânula metálica retirar a cânula interna e lavar. Se nenhuma destas manobras funcionar, chamar ajuda para troca de cânula ou intubação orotraqueal ${ }^{17}$.
Quanto à frequência de aspiração da cânula traqueal, não houve consenso também entre os especialistas. Na literatura, o consenso é que não há um protocolo de periodicidade de aspiração, pois dependerá da quantidade e fluidez da secreção eliminada por cada paciente. Pacientes com bom reflexo de tosse necessitam de menos aspirações durante o dia. Na série de questionários apresentada aos nossos especialistas, obtivemos uma resposta semelhante, de individualizar a frequência de aspiração para cada paciente. A frequência de limpeza da cânula interna segue a mesma teoria de avaliar o paciente para definir a frequência ${ }^{17}$.

O tempo mínimo necessário para que seja realizada a primeira troca da cânula não foi definido pelos especialistas da FCECON. Protocolos já estabelecidos recomendam que seja feita a primeira troca da cânula, em casos de necessidade, entre 48 e 72 horas no mínimo ${ }^{18,19}$. As trocas periódicas das cânulas metálica e de plástico com balonete também não obtiveram consenso, sendo que está recomendado que sejam trocadas a cada 30 dias (metálica) e entre sete e 14 dias (plástico) ${ }^{18,19}$. Estes dados são fundamentais nas condutas de enfermaria, UTI e ambulatório.

Em relação a um protocolo de decanulação também não houve consenso. Analisando os comentários relatados pelos especialistas, procede a sugestão de não determinar um protocolo de decanulação e sim personalizar de acordo com o motivo da traqueostomia e comorbidades associadas.

Não houve consenso sobre qual seria o profissional da equipe que iria realizar o treinamento para os cuidadores de pacientes com traqueostomia. O relato dos especialistas nas observações foi que cada profissional fizesse a orientação da sua parte. A literatura relata orientação semelhante, descrevendo a importância 
da orientação de todos os profissionais da equipe em relação aos cuidados com a traqueostomia e não um único profissional20,21.

Ao ser efetivado na FCECON, espera-se que $\mathrm{o}$ protocolo promova um atendimento multidisciplinar padronizado adaptado às particularidades dos pacientes. Esta forma de atendimento permite que eventuais mudanças no quadro de profissionais não modifiquem $\mathrm{o}$ modelo de tratamento. Além do atendimento personalizado, os pacientes e seus cuidadores serão orientados quanto à assistência e cuidados no domicílio. Consultar os especialistas na nossa região que trabalham na FCECON foi a opção para essa adaptação, pois conhecem a rotina dos pacientes. É sabido que existirão casos particulares em que será necessário que o especialista personalize a conduta. Ao realizar a revisão deste protocolo, anualmente ou a cada biênio, sugere-se tentar convocar um maior número de profissionais e principalmente convocar membros de outras especialidades que não participaram da elaboração deste texto inicial, podendo assim, elaborar um manual mais amplo em relação ao conceito de multidisciplinaridade, tão importante para o paciente que é submetido a uma traqueostomia.

\section{A B S T R A C T}

Objective: to create a multidisciplinary conducts manual for tracheostomies in adult and pediatric patients in the Amazonas State Oncology Control Center Foundation. Methods: we developed a protocol using the modified Delphi method, which consisted in the application of two series of questionnaires to 20 professionals of the unit. Results: thirteen professionals completed the two steps. In the first stage, there was consensus in 53 out of 92 questions (57.6\%). The questions that obtained consensus formed the text of the second stage, divided into eight chapters and evaluated by marking the answers offered on a Linkert scale. All the chapters presented in the second stage obtained consensus, meaning that the sum of the answers "agree" and "fully agree" were above $70 \%$. Conclusion: using the data obtained in the consensus, we elaborated a tracheostomy conduct protocol and a care guidelines manual for the patients and their caregivers.

Keywords: Clinical Protocols. Tracheostomy. Medical Oncology. Hospitals. University.

\section{REFERÊNCIAS}

1. Ricz HMA, Mello Filho FV, Freitas LCC, Mamede RCM. Traqueostomia. Medicina (Ribeirão Preto). 2011;44(1):63-9.

2. Hammarfjord O, Ekanayake K, Norton J, Stassen LF. Limited dissection and early primary closure of the tracheostomy stoma in head and neck oncology operations: a retrospective study of 158 cases. Int J Oral Maxillofac Surg. 2015;44(3):297-300.

3. Coyle MJ, Shrimpton A, Perkins C, Fasanmade A, Godden D. First do no harm: should routine tracheostomy after oral and maxillofacial oncological operations be abandoned? $\mathrm{Br} J$ Oral Maxillofac Surg. 2012;50(8):732-5.
4. Salgarelli $A C$, Collini $M$, Bellini $P$, Capparè $P$. Tracheostomy in maxillofacial surgery: a simple and safe technique for residents in training. J Craniofac Surg. 2011;22(1):243-6.

5. Mogedas-Vegara A, Bescós-Atín C, GutiérrezSantamaría J, Masià-Gridilla J, Pamias-Romero J, Sáez-Barba M. Manejo de la vía aérea en oncología de cabeza y cuello. Rev Esp Cir Oral Maxilofac. 2014;36(4):164-8.

6. Scott N, Bater M, Fardy M. Tracheostomy in head and neck oncology. Results of the 2014 Tracheostomy Survey of the BAOMS Oncology Specialist Interest Group. Br J Oral Maxillofac Surg. 2015;53(8):779-81. 
7. Mitchell R, Parker V, Giles M. An interprofessional team approach to tracheostomy care: a mixedmethod investigation into the mechanisms explaining tracheostomy team effectiveness. Int J Nurs Stud. 2013;50(4):536-42.

8. Hasson F, Keeney S, McKenna H. Research guidelines for the Delphi survey technique. J Adv Nurs. 2000;32(4):1008-15.

9. Hsu CC, Sandford B. The Delphi technique: making sense of consensus. Pract Assess Res Eval (Online). 2007;12(10):1-8.

10. Mitchell RB, Hussey HM, Setzen G, Jacobs IN, Nussenbaum B, Dawson $C$, et al. Clinical consensus statement: tracheostomy care. Otolaryngol Head Neck Surg. 2013;148(1):6-20.

11. Urrestarazu P, Varón J, Rodríguez A, Ton V, Vila $F$, Cipriani $S$, et al. Consenso sobre el cuidado del niño con traqueostomía. Arch Argentina Pediatr. 2016;114(1):89-95.

12. Patel $S A$, Plowman EK, Halum S, Merati AL, Sardesai MG. Late tracheotomy is associated with higher morbidity and mortality in mechanically ventilated patients. Laryngoscope. 2015;125(9):2134-8.

13. Liu CC, Livingstone D, Dixon E, Dort JC. Early versus late tracheostomy: a systematic review and meta-analysis. Otolaryngol Head Neck Surg. 2015;152(2):219-27.

14. Scurry WC Jr, McGinn JD. Operative tracheotomy. Oper Tech Otolaryngol Head Neck Surg. 2007;18(2):85-9.

15. Durbin CG Jr. Tracheostomy: why, when, and how? Respir Care. 2010;55(8):1056-68.
16. Fraga JC, Souza JCK, Kruel J. Traqueostomia na criança. J Pediatr (Rio J). 2009;85(2):97-103.

17. Singapore. Ministry of Health. Moh Nursing Clinical Practice Guidelines 2/2010. Nursing Management of Adult Patients with Tracheostomy. Ministry of Health: Singapore, 2010.

18. Dawson D. Essential principles: tracheostomy care in the adult patient. Nurs Crit Care. 2014;19(2):63-72.

19. National Tracheostomy Safety Project Manual [Internet]. Manchester: National Tracheostomy Safety Project; 2013. Availbale from: www. tracheostomy.org.uk

20. McCormick ME, Ward E, Roberson DW, Shah RK, Stachler RJ, Brenner MJ. Life after tracheostomy: patient and family perspectives on teaching, transitions, and multidisciplinary teams. Otolaryngol Head Neck Surg. 2015;153(6):914-20.

21. Yelverton JC, Nguyen JH, Wan W, Kenerson MC, Schuman TA. Effectiveness of a standardized education process for tracheostomy care. Laryngoscope. 2015;125(2):342-7.

Recebido em: 30/01/2018

Aceito para publicação em: 10/05/2018

Conflito de interesse: nenhum.

Fonte de financiamento: nenhuma.

\section{Endereço para correspondência:}

Maria Carolina Coutinho Xavier Soares

E-mail: mariaccxs@hotmail.com

mcsoares@uea.edu.br 